Théologiques

Théologiques

\title{
Grégoire de Nazianze. Le miroir de l'Intelligence ou le dialogue avec la Lumière
}

\section{Francis Gautier}

Volume 16, numéro 2, 2008

L’individualisation de la relation religieuse

URI : https://id.erudit.org/iderudit/001633ar

DOI : https://doi.org/10.7202/001633ar

Aller au sommaire du numéro

\section{Éditeur(s)}

Faculté de théologie et de sciences des religions, Université de Montréal

ISSN

1188-7109 (imprimé)

1492-1413 (numérique)

Découvrir la revue

Citer cet article

Gautier, F. (2008). Grégoire de Nazianze. Le miroir de l'Intelligence ou le dialogue avec la Lumière. Théologiques, 16(2), 31-47.

https://doi.org/10.7202/001633ar
Résumé de l'article

Grégoire de Nazianze, le plus affirmatif des Cappadociens quant à la divinisation de l'homme dès cette vie, semble logiquement minorer le fossé entre la vision de Dieu " comme en un miroir " ici-bas et le " face à face " promis (1 Co 13,12). Cet article examine ce fait par l'analyse des métaphores optiques utilisées par Grégoire pour les relations entre la « Lumière » ou l'Intellect divin et son " image ", l'homme, aux différentes étapes de l'économie du salut. Le Nazianzène situe la relation de l'individu à Dieu, dynamique et assimilative, entre la « Lumière »/Intelligence divine et son reflet émané en nous. Leur union en Christ rétablit à travers l'illumination baptismale la translucidité adamique perdue, rouvrant à l'individu le passage de la vision " en miroir » d'ici-bas de sa source, l'essence divine, à l'illumination plénière $\mathrm{du}$ « face à face » divinisateur. L'imagerie nazianzène du " dialogue " contemplatif entre le nous ou " lumière " humain(e) et Dieu, quelle que soit l'insistance sur la transcendance du second à l'égard du visible et sur les attaches du premier au sensible, implique réellement pour le nous contemplatif une communication avec, et une assimilation à la source dont il émana. L'intellect-miroir du contemplatif, à condition d'un "nettoyage " portant à la perfection noétique les images reflétant Dieu, peut déjà en former un aperçu grâce à la lumière de l'Esprit, en recevoir les " énergies », et servir de médiateur sacerdotal entre la Lumière et les autres individus.
Ce document est protégé par la loi sur le droit d'auteur. L'utilisation des services d'Érudit (y compris la reproduction) est assujettie à sa politique d'utilisation que vous pouvez consulter en ligne.

https://apropos.erudit.org/fr/usagers/politique-dutilisation/ 


\title{
Grégoire de Nazianze. Le miroir de l'Intelligence ou le dialogue avec la Lumière
}

\author{
Francis GAUTIER" \\ Philosophie \\ École Pratique des Hautes Études
}

Comme l'a montré Donald F. Winslow, la "divinisation ", theôsis, recouvre chez Grégoire de Nazianze une relation assimilative à Dieu qui embrasse toute l'économie divine, de la création d'Adam à la résurrection. Parmi les registres métaphoriques exprimant cette "relation dynamique " selon Winslow (1979, 187-198), celui, visuel, de la lumière, nous paraît décliner et résumer tous les autres: l'épistémologique dont — comme pour toute la pensée grecque - il est le modèle; celui, spatial, de l'ascension; l'éthique; celui de l'union ou du mélange avec Dieu; ou celui, social, de l'intimité avec lui. Il exprime comme «dialogue » de la lumière humaine avec la Lumière divine ${ }^{1}$ une relation internoétique conduisant notre intelligence de la vision «en un miroir» de l'Intelligence au «face à face» (1 Co 13,12); et ce, en puisant dans la riche thématique scripturaire de la lumière, interprétée d'après la noétique antique et ses modèles optiques.

Quelques rappels sur les théories et le lexique optiques anciens sont donc indispensables. Selon le modèle optique le plus courant, platonicien,

\footnotetext{
* Francis Gautier est professeur certifié de philosophie à l'École Pratique des Hautes Études de Paris. Il est actuellement affecté au service des publications et donne un enseignement de patristique grecque en première année de Master. Spécialiste de Grégoire de Nazianze, sur lequel il poursuit ses recherches en tant que membre du Laboratoire d'Études des monothéismes (UMR 8584), il a publié en 2002 La retraite et le sacerdoce chez Grégoire de Nazianze (Bibliothèque de l'École des Hautes Études, Sciences religieuses 114, Turnhout, Brepols).

1. Expression que nous suggère Richard (2003, 116-117).
} 
l'œil, «semblable au soleil » (hêlioeidês), émet un flux igné qui s'unit à la lumière solaire par sa "parenté » avec elle ${ }^{2}$, atteint ainsi les objets qu'elle éclaire et en reçoit les impressions ${ }^{3}$. L'inégalité entre la lumière visuelle et celle du soleil, visible supérieur, nous éblouissant, nous ne pouvons voir que le reflet de celui-ci ${ }^{4}$. Si ce modèle fait procéder la vision catoptrique d'un rebond du flux visuel, le « reflet ", emphasis, est surtout conçu comme «empreinte» (tupos) du visible dans le miroir, et la réflexion de la lumière peut être considérée comme une émanation seconde du reflet de sa source. Les miroirs antiques, de métal poli, peu fidèles et soumis aux ternissures de la corrosion, relevaient pourtant du "diaphane », nature de certains milieux, tels l'éther, l'air ou l'eau, apte à recevoir la lumière et faire ainsi transparaître le visible. Cette catégorie commande l'autre grand modèle optique, aristotélicien, qui définit la lumière comme être en acte du milieu diaphane actualisé par un illuminant et dès lors médiateur, des visibles à l'œil, d'une sorte d'impulsion qui produit la sensation visuelle (Vasiliu 1997, 39-63 et 162-195 ; 2004, 307-309). Plotin récuse tout rôle positif du milieu diaphane dans la vision pour faire de la lumière un acte, ou une énergie intérieure, du corps lumineux, principe de l'acte second de la lumière qui en émane et qui est elle-même le milieu immatériel d'une vision par sympathie ${ }^{5}$.

Phôs désigne tant la lumière que sa source, le "luminaire». Ses dérivés phantazômai et phantasia, rendus le plus souvent par «imaginer» et «imagination» selon leur connotation déceptive chez Platon, désignent chez Aristote l'action et la faculté de former, par la perception ou par dérivation, des représentations - phantasmata - vraies ou fausses, ainsi que ces représentations elles-mêmes. Les stoïciens n'en retinrent que le sens positif, réservant phantasma à l'imaginaire, l'illusoire. L'eidos ou l'idea, c'est la vue de ce qui est, sa forme véridique; eidôlon, son double apparent et trompeur, d'où sa traduction usuelle par «simulacre» et son sens d' "idôles » dans

2. République, $508 \mathrm{~b}$ et e; Timée, $45 \mathrm{~b}-46 \mathrm{a}$.

3. Voir Merker (2003, 24-39); Simon (1988, 22-38); Diehle (1983, 85-91). Brisson (1974, 440 et 446) privilégie le moment proprement perceptif de la rencontre entre les «feux » émanant des objets et de la vue (Timée, 67c; Thééthète, 156d-e), mais Timée, 45a-46a et République, 507d-509b insistent sur le rôle de la lumière du jour qui les «conjugue ", "entoure» ou "enveloppe » le flux visuel.

4. République, 515c-516a; 518a-d; Timée, 67e-69a.

5. Ennéades, Iv 5. 
les LXx (2 R 17,12); eikôn, l'image douée de similitude ${ }^{6}$, ainsi en Gn 1,26 pour la création de l'homme "à l'image » et en Col 1,15 du Christ "Image du Dieu invisible». Eikasia signifie "la façon dont on se figure une chose, représentation imagée ou conjecture ». Skia, l' "ombre », mais, pour la lumière, son reflet lumineux, désigne chez les chrétiens les préfigurations prophétiques de l'Ancien Testament.

Pour le Nazianzène, «Dieu est lumière » (Jn 1,4-5.9; 8,12) du monde intelligible en tant qu' "Intellect (Nous) premier" ", et il lui applique à ce titre l'analogie de la République (vi, 19, 507d-508e) entre le soleil et le Bien :

L'un en effet éclaire le monde visible, l'autre l'invisible; et l'un rend les yeux corporels semblables au soleil (hêlioeideis), l'autre les natures intellectives semblables à Dieu (theoeideis). Et comme le premier, procurant la puissance de voir à ceux qui voient et aux visibles la possibilité d'être vus est lui-même le plus beau des visibles, de même, Dieu, qui procure l'intellection aux intelligences et l'intelligibilité aux intelligibles, est lui-même le plus élevé des intelligibles où toutes choses arrêtent leur élan [...]. Car c'est là le terme des désirs et le repos d'une contemplation totale pour ceux qui en sont arrivés là̊

Les parallèles abondent avec les Ennéades ${ }^{9}$, mais elles situent le Bien audelà de l'intelligible et de l'Intellect qui en émane comme la lumière émane du soleil, tout en admettant une vision intuitive du Bien quand Grégoire réserve la contemplation de Dieu à l'au-delà. Grégoire est en cela plus proche de la tradition aristotélicienne, selon laquelle les intellects n'actualisent leur potentialité noétique qu'illuminés par le Nous/Bien divin comme

6. Sur ces définitions, voir Simon (2004, 335-340); Labarrière (2004, 931-935); Frère (1996).

7. D. 18,4. Voir Moreschini (1997, 32, 45-47, 69-70, 73-76, 99-100).

8. D. 21,1 (Sources Chrétiennes [ci-après abrégé SC] 270, trad. Mossay, 112-113, retouchée). Voir aussi D. 28,30.

9. L'Intellect, "soleil des intelligibles » (IV 3,11,14-15) dont la lumière «éclaire l'âme en l'illuminant et la rend intelligente en la faisant semblable à elle-même » (v 3,8,2426); l’Un/Bien analogue du soleil, l'Intellect de sa lumière (v 1,6,19 sqq.; 3,12,1-28) qui confère "l'être intelligéll'intelliger [...] aux étants et à l'intellect » analogues des visibles et de ce qui voit (vi 7,16,24 sqq.); la finalité de l'âme est de voir l'Un/Bien source de sa lumière ( $\mathrm{v} 3,17,33$ sqq.), ce qui porte l'intellect à sa plénitude (III 8,11,6); le Bien nous attire et nous trouvons en lui le repos (vi 7,23,1-9). Les références et traductions des Ennéades sont données d'après l'édition de Bréhier. 
le diaphane ne s'actualise que sous l'action d'un illuminant igné ou du «corps supérieur » éthéré, diaphane en acte/lumineux par soi ${ }^{10}$.

Dieu crée anges et hommes, "nature intelligente ", comme des «miroirs translucides (diaugea $\approx$ diaphanea) de la Lumière primordiale » sur lesquels il « répand la divinité dont il est la source ${ }^{11}$ ». Cette image dit leur participation mimétique et leur dépendance ontologique à l'égard de l'Intelligence qu'elles reflètent et réfléchissent, Grégoire opposant « la lumière qui illumine, phôs phôtistikon, à la luminosité causée, phôs phôtistikon echôn » (Richard 2003, 113, voir n. 12). Selon la lecture nazianzène de Gn 2,7, suggérée par rapprochement avec Sg 7 (vv. 7.10.22-27), Dieu a uni en Adam le visible et l'intelligible ${ }^{12}$ en insufflant dans son corps un "souffle du grand Intellect ${ }^{13}$ ", "c'est-à-dire une âme intellective et une image de Dieu $^{14}$ », ou en y instillant une "goutte (aporrôx) de la lumière infinie de la divinité ${ }^{15} »$. Ces images expriment le don originel d'une participation au Nous divin constituant l'homme à son image (Winslow 1979, 51; Richard 2003, 26 et 263-264) — pour autant qu'en lui « la nature de l'intelligence domine ${ }^{16} »-$, troisième lumière après Dieu et les anges ${ }^{17}$, et, comme ces

10. Alexandre d'Aphrodise, De Anima, 87,26-90,20, identifiant «l'intellect agent» d'Aristote au Premier intelligible/Intellect suprême de Métaphysique L, actuel par soi, en fait la «cause de l'intellection des autres intelligibles » par sa «venue " en nous quand nous le pensons et que nous nous assimilons ainsi à lui, comme le "luminaire ", "visible par excellence ", actualise les autres visibles — cf. 42,19—46,6, en actualisant, en tant que diaphane actuel/lumineux par soi, le milieu diaphane. Pour récuser l'émanation matérielle et locale de la lumière, 42,19—43,12 compare l'illumination du diaphane par le luminaire à la production du reflet dans un miroir par sa mise en présence de, ou en relation avec l'objet reflété, comparaison reprise et appliquée par Plotin aux relations des âmes à l'Âme dont elles dépendent comme «acte subordonné » (Iv 5,7,33-50). Thémistius, De Anima Paraphrasis, 59,10-30, distingue de la lumière que le soleil procure aux autres diaphanes en les actualisant l'entéléchie propre de son être diaphane. Il compare, 98,12-99,10, ce rapport hiérarchique à celui de l'Intellect divin aux intellects potentiels, ses «précurseur[s] » dans les corps «comme le rayon de soleil pour la lumière du jour» $(105,30)$, et l'actualisation par l'Intellect des intellects et des intelligibles potentiels à celle de la vue et des couleurs par la lumière (voir Vasiliu 1997, 80-103).

11. P. $1,1,4,77-81$.

12. D. $38,10-11 ; 2,75$; P. $1,1,8,57-77 ; 1,2,1,79-99 ; 1,2,10,98-116$.

13. P. $1,2,12,8$.

14. D. 38,11 (SC 358,124$)$.

15. P. $1,1,4,32-33$. Voir $1,1,8,73=1,2,1,95$; D. 32,15 ; P. 1,2,10,60. Voir Clément d'Alexandrie, Protreptique 6, cité dans Moreschini (1997, 25).

16. P. $1,1,8,75-76=1,2,1,97-98$.

17. D. 6,12 ; D. 28,31 ; 38,9; P. $1,1,7,8-13$; $1,2,9,68-69$; 2,1,45,287-290. 
derniers, "apparenté » (oikeios) à Dieu ${ }^{18}$. L'inégalité entre le pur intellect angélique et l'humain, mêlé à la matière, tient à la réceptivité à la Lumière divine - la "diaphanéité » noétique - de leurs natures, respectivement comparées à l'éther et à l'air dans leur rapport au soleil ${ }^{19}$. Grégoire suit là l'idée platonicienne selon laquelle le milieu où se forme l'image détermine sa différence d'avec son modèle (Brisson 1974, 195-196).

Le «penchant vers Dieu ${ }^{20}$ » inscrit en Adam par affinité tient son nous dans un rapport exclusif avec la Lumière qui préserve son hégémonie unitive sur la nature composite de la créature qu'il mène vers sa divinisation (Althaus 1972, 13-15, 57, 72-80 ; Richard 2003, 115, 343-346, 371-373, 477). Mais par le péché originel, son intellect, se détournant de Dieu, rompt cette relation et perd cette hégémonie (Althaus 1972, 34-36, 85-88; Winslow 1979, 53, 68; Richard 2003, 271, 288). Par contrecoup, son corps revêt les «tuniques de peau » $(\mathrm{Gn} 3,2)$, « une chair épaissie, mortelle et rebelle " qui tire le nous vers le sensible et le rend opaque à la Lumière divine $^{21}$.

Un reste de lumière naturelle permet de reconnaître l'existence de Dieu à partir du reflet de sa gloire dans la création, soit la vision de Dieu «par derrière » d'Ex 33,23 (Richard 2003, 35-37, 46-47). Mais c'est grâce à l'Incarnation et par le baptême, dit aussi «illumination ${ }^{22}$ », que l'individu renoue avec la Lumière divine.

L'Incarnation se fait par l'union des intellects humain et divin qu'autorise leur "parentée ${ }^{23} »$ : le Christ, individu archétypal de notre divinisation, est «tellement "visité d'en haut par le soleil levant" (voir Lc 1,78) », qu'il devient Dieu ${ }^{24}$. Ce «Mélange» ou cette «fusion ${ }^{25}$ » n'abolit pas le nous humain - auquel Apollinaire substituait le Verbe -, mais l'assume pour son salut (Winslow 1979, 79-95; Gallay 1974, 16-19): "Mais où donc sera

18. D. 2,$23 ; 28,17$; Ep. 101,49 ; P. $1,1,10,35$.

19. P. $1,1,7,50-52$; Richard $(2003,138,143-149)$.

20. D. 38,$11 ; 44,4$; P. $1,1,8,97-99 ; 2,1,45,10$.

21. Richard 2003, 260-262, 278-283, 288 ; Szymusiak 1966, 288-305. Voir Athanase, Contre les païns 8,16d,3-10: "détournée du bien et oubliant qu'elle est l'image du Dieu bon, la puissance qui est en [l'âme] ne voit plus le Dieu Verbe, à la ressemblance de qui elle a été faite [...] car elle a caché dans les replis des désirs corporels le miroir qui est en elle, par lequel seul elle pouvait voir l'Image du Père », trad. Camelot (SC 18bis, 73).

22. D. 40,3 et $36-37$.

23. D. 29,$19 ; 38,13$; Ep. $101,36-39$ et 49.

24. D. 30,3. Voir Ennéades v 5,8,5-7.

25. D. 2,23 ; 30,2-3 ; 34,10 ; 37,2 ; 38,13 ; Ep. 101,39-40 ; P. 1,1,2,57 s.; 2,1,11,612. 
l'accomplissement de l'intellect de l'ange ou de l'homme par la fusion avec la divinité, si par la présence du plus grand [le Nous divin] l'autre est éliminé ? " Il en va comme du rayon présent au sein de la lumière solaire dans la maison de l'âme ${ }^{26}$ : en Christ, « la nature humaine a reçu en partage Dieu tout entier,/ non comme un prophète ou un des inspirés de Dieu (entheôn)/ - qui participe non de Dieu, mais de ce qui est de Dieu -, mais substantiellement, comme le soleil pour ses rayons ${ }^{27}{ }$. Tant la lecture de Lc 1,78 que ces dernières images font écho aux descriptions de la vision du Bien chez Plotin, mais pour dire la présence plénière de Dieu en $\mathrm{Christ}^{28}$. Le Verbe porte notre humanité pour y «consumer» le péché comme "le soleil la brume de la terre ». Dieu rendu visible, homme exempt du péché et soumis au vouloir divin, le Christ offre un modèle de vie divin ${ }^{29}$ dont il faut peindre sur soi la « vertu merveilleuse ${ }^{30}$ ».

Dit aussi «illumination ${ }^{31}$ ", le baptême, appropriation individuelle, par la grâce de l'Esprit, de la rédemption générique opérée en Christ (Winslow 1979, 129; Richard 2003, 470), "dégage des passions en ôtant le voile qui nous enveloppe depuis que nous sommes engendrés et en nous ramenant vers la vie d'en haut »; il restaure la translucidité adamique, est " participation de la Lumière ", "disparition des ténèbres ", "perfection de l'intelligence ${ }^{32} »$. Mais seule une élite purifiée de corps, d'âme et d'intellect peut contempler la pureté de la Lumière divine ${ }^{33}$ et dépasser les lumières de la foi baptismale: les moines, "lumières du monde, miroirs translucides de la Lumière ${ }^{34}$ ». Ils se gardent des fausses illuminations, ténèbres qui se donnent pour « la lampe [Dieu] de notre principe directeur [le nous] (voir $\operatorname{Pr} 20,27$; Ps 18,29) qui conduit droit nos pas selon Dieu (cf. Ps 119,105) »,

26. Ep. 101,40 (SC 208, 52); voir P. 2,1,11,642-645. Voir Plotin, Ennéades v 3, 17, 28 ; Thémistius, De anima Paraphrasis 105,30 (voir n. 12).

27. P. 2,1,11,642-645. Voir Origène, Traité des principes, II,6,6 (SC 252, 199-200): «le feu divin lui-même repose substantiellement " dans l'âme du Christ.

28. Voir n. 32 et 35 . Ennéades v 5,10,5-7 précise que nous ne pouvons saisir qu'en partie la puissance du Bien, sans quoi nous n'en différerions pas.

29. D. 30,5-6.

30. D. 2, 13. Voir D. 38,18.

31. D. 40,3 et $36-37$.

32. D. 40,3-4, trad. C. Moreschini (SC 358, 201-203).

33. D. 2,$39 ; 76 ; 20,4 ; 27,3 ; 39,9 ; 32,15 ;$ P. $2,2,7,221$.

34. P. $1,2,1,213$. Voir P. 1,1,4,78. 
par un contrôle du corps et de l'intentionnalité : « ne laissons rien qui ne soit illuminé. Purifions l'œil, afin que notre regard soit droit (cf. Mt 6,22), et ne portons en nous-mêmes aucune idole ${ }^{35} »$ - bien ou être sensible, ou notion de Dieu prise pour Lui ${ }^{36}$. La pureté d'âme exige l'ascèse morale, sociale: la "pratique ", "marchepied » ou "procureur» de la contemplation ${ }^{37}$, chaque étape dans l'illumination produisant par assimilation un degré de vertu plus éclatant, base pour un nouveau progrès contemplatif ${ }^{38}$. Devenir lumière ou flambeau pour les autres ${ }^{39}$, surtout dans l'autorité théologique de l'évêque, «lampe » de l'Église ${ }^{40}$, requiert cette double ascèse, le sacerdoce d'avoir obtenu participation à «toutes les appellations et les puissances du Christ» — dont celles du Verbe — « et par l'action et par la contempla$\operatorname{tion}^{41} »$.

La «familiarité » (oikeiôsis) contemplative avec Dieu ${ }^{42}$ relève du même registre lexical de la «maisonnée » (oikos) que la "parenté » entre Intellect et êtres intelligents. Proche de la filiation adoptive $e^{43}$ - par opposition à celle du Fils, Image identique et consubstantielle au Père ${ }^{44}$-, ce lexique est relayé par celui du «commerce» ou de la «conversation» avec Dieu ${ }^{45}$ du moine «ami de Dieu" ${ }^{46}$ » (voir Ex 33,11; 34,29): un dialogue contemplatif avec la Lumière divine dans lequel l'intellect « est et devient toujours plus un miroir immaculé de Dieu (voir Sg 7,26; 2 Co 3,18) et des réalités divines en adjoignant la Lumière à [sa] lumière ${ }^{47}$ ».

Or, Grégoire reprend souvent l'opposition de 1 Co 13,12 entre la vision de Dieu dans un miroir accessible ici-bas, obscure et partielle, et le face à face promis formulé, d'après Platon, comme «contact direct, à nu » de

35. D. 40,37-38 (SC 358, 282-284).

36. Ce que Børtness $(2006,45)$ appelle «idolâtrie conceptuelle ».

37. D. 4,$113 ; 20,12 ; 40,37$.

38. D. 21,6; 24,15. Voir Moreschini $(1997,100)$; Gautier (2002, 99-100).

39. D. 2,$71 ; 12,4 ; 25,11 ; 40,37$.

40. Voir D. 2,72; 10,3,16; Ep. 41.

41. D. 2,98. Voir D. 39,$10 ;$ P. 2,1,1,264; P. 1,2,1,211.

42. D. 2,3 et $17 ; 21,2 ; 32,15 ; 38,7$.

43. Voir P. 1,2,10,140-143.

44. D. 30,$20 ; 38,13=45,9 ;$ P. $1,1,2,8 ; 1,2,1,145-146 ; 2,1,1,628 ; 2,1,45,32$.

45. D. 2,3 et $7 ; 9,1 ; 10,1 ; 12,4 ; 20,1$ et $4 ; 21,2$ et $19 ; 32,15 ; 38,7$; P. $2,1,11,353$; $2,1,12,808$.

46. D. 21,6 ; P. $2,1,1,611 ; 2,1,11,323$.

47. D. $2,7=20,1$. 
notre intellect avec l'Intellect divin (Richard 2003, 148) $)^{48}$. Parvenus auprès de sa source, gravitant alentour comme une "lumière inférieure ", nous serons alors unis ou mêlés à la Lumière, recevant tout son rayonnement (Winslow 1979, 172). Mais nous resterons distincts de sa source, la créature ne devenant "dieu» qu'au sens participatif d'un état angélique (Winslow 1979, 186-187; Richard 2003, 336, 477; Russell 2006, 225). Le corps ressuscité, spiritualisé, ne gêne plus la vision de Dieu ${ }^{49}$ — les miroirs étant dissous par la Vérité ${ }^{50}$-, mais individue les créatures par rapport à lui et entre elles (Winslow 1979, 175-176). Les distinctions de la chair abolies car nous portons «en nous-mêmes seulement l'empreinte divine (cf. 2 Co 1,22) ${ }^{51}$ ", celle, spirituelle, des demeures célestes s'y substitue ${ }^{52}$. Leur hiérarchie répond à l'échelle des charismes, dont le plus haut est théologique, car « la rétribution [...] c'est la lumière pour ceux qui ont opéré la purification de leur pensée (tên dianoian), c'est-à-dire Dieu vu et connu à proportion de la puretés3 $"$.

Même quand il se réfère à 1 Co 13,12, Grégoire attribue au contemplatif, en vertu de la pureté du nous-miroir et des reflets divins qu'il forme, des traits anticipant le salut: souveraineté ou extase du nous, proximité de Dieu ou isangélie, contemplation de $\mathrm{Dieu}^{54}$, grâce de «se mêler à la pure Lumière » pour avoir surmonté «la dualité relative à la matière par l'unité intelligée dans la Trinité 55 ». Il lui accorde même, non sans précautions oratoires, une anticipation du face à face divinisateur :

La divinité $[\ldots]$ en purifiant $[\ldots]$ rend aussi semblable à Dieu; avec ceux qui sont devenus tels, il s'entretient alors comme avec des familiers; et - je parle ici avec une certaine audace - Dieu à des dieux (cf. Ps 81, 1.6) s'unit et en est connu, et peut-être autant que lui-même connaît déjà ceux qui sont connus de lui (cf. 1 Co 13,12$)^{56}$.

48. D. 7,21 attribue ce «contact » à l'état séparé de l'âme sauvée avant la résurrection, après laquelle l'âme y fait participer la chair car "elle l'a tout entière assimilée en ellemême et ne fait qu'un avec elle: un seul esprit, une seule intelligence, un seul dieu ", trad. Calvet-Sébasti (SC 405, 235).

49. D. 7,21; 14,6; A. Richard 2003, 284-291.

50. D. 12,4 .

51. D. 7,23, trad. Calvet-Sébasti (SC 405, 240).

52. D. 30,4 .

53. D. 40,45, trad. Moreschini (SC 358,307). Voir D. 27,8; 32,11-13.

54. D. $2,7=20,1 ; 12,4 ;$ P. $1,2,1,209-214 ; 2,1,1,194-211 ; 2,1,36,7-10$.

55. D. 21,2, trad. Mossay (SC 270, 112-114).

56. D. 38,7, trad. Moreschini (SC 358, 117); voir P. 1,2,1,214. 
Cette relativisation de l'opposition paulinienne n'est pas que rhétorique: la continuité dynamique et proportionnelle entre l'illumination d'ici et l'eschatologique ramène leur différence à une distance noétique que minore l'infinité de la visée contemplative ${ }^{57}$.

Pour le comprendre, examinons les difficultés et les limites de la gnose d'ici-bas dans les Discours théologiques. Ce, sans oublier quelles cibles ils visaient: le littéralisme opposé au «consubstantiel » nicéen et à la confession de l'Esprit comme Dieu et le rationalisme nominaliste d'Eunome, qui estimait Dieu connaissable par la seule application de notre intelligence aux Écritures et - contre la divinité du Fils - prétendait le définir en son essence comme «inengendré » (Norris 1991, 61 sqq.; McGuckin 2001, 283-289; Børnstein 2006, 44-45).

L'opacité que le corps impose à l'intellect — « ténèbres » d'Ex 10,22 ou "obscurité » de Ps 17,12 —, soit la médiation de l'imagination, issue du sensible, par laquelle, selon l'aristotélisme, il accède aux intelligibles, semble exclure une relation noétique pure, donc adéquate, à Dieu :

De même que la vue ne peut pas accéder aux choses visibles sans la lumière et l'air intermédiaires [...] il n'y a pas moyen pour ceux qui sont dans un corps d'être entièrement avec les réalités intelligibles sans les réalités corporelles. Toujours en effet s'insinuera quelque chose de ce qui est nôtre, même si l'intellect, dégagé le plus possible des choses visibles et devenu ce qu'il est par lui-même, essaie de s'appliquer aux choses qui lui sont apparentées et qui sont invisibles ${ }^{58}$.

Même s'il saisit leur sens spirituel, notre nous est tributaire des images qu'évoquent les noms scripturaires de Dieu. Il peine à concevoir dans leur perfection les attributs divins qu'elles figurent et à en approcher, par la synthèse, l'unité essentielle:

57. D. 12,$4 ; 21,1-2 ; 32,15 ; 40,45 ;$ P. 1,2,1,209-214.

58. D. 28,12, trad. Gallay (SC 250, 125-127). Voir Aristote, De anima II,7,418b-419a, la vision exige un milieu diaphane; III,7,431a-b, «l'âme ne pense jamais sans image », «la faculté noétique pense [...] les formes dans les images », dérivées de la sensation $(3,428 b-429 a) ; 6,430 a$, «c'est seulement quand [l'intellect agent] est séparé qu'il n'est que ce qu'il est en propre ». Selon Alexandre d'Aphrodise, l'intellect progresse de la contemplation des formes intelligibles qu'il a abstrait de la phantasia à celle des intelligibles par soi/intelligences, immatériels et éternels, voir Schroeder et Todd (1990, 17-19, 62-64). Thémistius, De anima paraphrasis, 113,14-115,9, précise que l' «intellect théorique» ne peut penser ces derniers «continuellement et perpétuellement ». Voir aussi 100,16-36: «ce que c'est que d'être moi » "vient de l'intellect agent », non de l'intellect patient lié au corps, à l'imagination et à la dianoia. 
Faut-il plutôt [...] voir la divinité elle-même à partir de ces choses, autant que possible, en composant une certaine représentation partielle (merikên tina phantasian. Cf. 1 Co 13,9 et 12b) à partir de nos comparaisons (eikasmatôn) ? Mais le moyen de le voir à partir d'elles sans les voir elles-mêmes ? Et comment est-il toutes ces choses et chacune de façon parfaite, l'Un par nature incomposé et incomparable? Ainsi notre esprit (nous) s'épuise à sortir des choses corporelles et à entrer en contact avec les incorporels dans leur nudité, tant qu'il considère avec la faiblesse qui est la sienne ce qui dépasse sa puissance $^{59}$.

Mais c'est de l'essence même de Dieu que Grégoire réserve la connaissance à l'au-delà ${ }^{60}:$ du fait non tant de son invisibilité de pur être noétique - partagée par les anges ${ }^{61}$ - que de son infinité, de son être sans forme intelligible (aneideos $)^{62}$. En quoi il est, comme chez Plotin, analogue à la lumière, cause sans forme (abstraction faite de celle que lui confère le corps céleste auquel elle est liée) de la vision des formes, et exige une vision d'un genre supérieur ${ }^{63}$. Cela n'exclut pas, aidé par la sagesse ou la puissance divine, la réception d'empreintes purement noétiques de la Vérité ${ }^{64}$, voire l'éclair fugace d'une illumination théophanique où l'on entrevoit, sans pouvoir la saisir, Son «image " (tês alêtheias indalma) ${ }^{65}$. Il s'agit, selon des conceptions néoplatoniciennes ${ }^{66}$, de l'intuition évanescente et indicible du Dieu anéidétique que reflète ou re-présente, de façon discrète et fragmentaire car imagée et langagière, la pensée discursive (dianoia) des attributs qui en sont la «périphérie» ou le rayonnement intelligible ${ }^{67}$ et que le nous syn-

59. D. 28,13, trad. Gallay (SC 250, 126-128; nous souligons).

60. D. $28,17-19$.

61. D. 28,$31 ; 38,10 ;$ P. $1,1,7,13$.

62. D. 37,$2 ; 38,10 ;$ P. $1,1,8,73=1,2,1,95$.

63. Ennéades v 5,6-7; vi 7,16-17; vi 7,32. Cp. D. 28,13: les noms divins «feu» et "lumière » doivent s'entendre d'un feu «sans forme propre» et d'une lumière "non mêlée à l'air et séparée de ce qui, pour ainsi dire, l'engendre et l'illumine », trad. Gallay (SC 250, 127).

64. D. $28,21$.

65. D. 38,7, voir 28,20. Voir aussi Thémistius, De anima Paraphrasis, 112,10, identifiant le Nous à la Vérité.

66. Voir Armstrong (1990a, 32-36 ; 1990b, 163-172); Hoffmann (1997, 364-367).

67. D. 38,7 , «ce qui est autour /[dit] de lui »;28,3, «ce qui est à l'extrémité et arrive jusqu'à nous» (voir Ennéades vi,8,18), et 38,9, «tout ce qui est dit être de Dieu ou autour de Dieu ». Voir Origène, Contre Celse, vi,65 ; Basile, Ep. 234, trad. Lossky (2006, 69): "Tout en affirmant que nous connaissons notre dieu dans ses énergies, nous ne promettons guère de l'approcher dans son essence même. Car si ces énergies descendent jusqu'à nous, son essence reste inaccessible. » 
thétise en un «tableau d'ombre » (skiagraphia $\left.{ }^{68}\right)$, une "image » (indalma) ou un « reflet projeté » (aposkiasma) de l'essence divine ${ }^{69}$.

Dans le néoplatonisme (Armstrong 1990d, 10-11; 1990c, 424-425, 428, 430), la transcendance divine ne disqualifie pas l'usage théologique de l'imagination. Selon Plotin, les images de celle-ci, phantasia matérielle, si elles n'en divergent pas, accompagnent « comme son reflet ou la faible lueur prolongeant une intense lumière » la phantasia noétique qui «voit le Bien par l'intermédiaire de l'Intellect, car il n'est pas tellement caché qu'il ne pénètre jusqu'à elle puisqu'il n'y a pas entre elle et lui de corps qui fasse obstacle ${ }^{70}$ ». C'est ainsi qu'à rebours, pour Clément d'Alexandrie, l'Écriture, " "ombre” (skia) de la Vérité ", comme il le dit de l'Incarnation, "ombre de la Lumière ", "n'est pas ténèbres, mais illumination " (Le Boulluec 2006, 364); et que pour Philon $(1963,139)$ l'âme rationnelle, remontant "par le visible à la contemplation de l'invisible ", voit "comme en un miroir » "l'esprit (nous) invisible déposé dans les mots". Encore faut-il, dit Grégoire, un esprit assez pur pour recevoir la lumière noétique de l'Esprit qui «brille à l'intérieur des lettres précieuses ${ }^{71}$ ». S'il dénonce la dianoia «épaisse et matérielle » des ariens, incapables de faire abstraction du temps quant à la "génération » du Fils ${ }^{72}$ et critique les comparaisons trinitaires traditionnelles pour en soustraire les traits inadéquats, il reconnaît aux images théologiques inspirées une part de vérité schématique, pur objet noétique de contemplation dont il veut transmettre l'illumination ${ }^{73}$. La brachylogie de cette "définition brève »: "L'intellect est la vue intérieure, non circonscrite (ou perigraphos)./ Son acte est intellection et impression./ Le logos est

68. D. 30,17 et 38,7. On a retenu jusqu'ici le sens d' « esquisse ", mais ces textes associent au caractère partiel ( $e k$ merous) de la connaissance terrestre de Dieu, selon 1 Co 13,9 et $12 \mathrm{~b}$, un caractère composite et illusoire relatif à l'Un/Tout et font donc allusion au Parménide, 165c-d où il désigne une peinture en «trompe-l'œil » qui suggère contours et relief par juxtaposition d'aplats de couleurs contrastées: de loin, elle présente une unité formelle qui fait illusion, mais de près se révèle composite et illusoire, voir Rouveret (1989, 13-62). L'image évoque la problématique de l'Un et du multiple du Parménide et des Ennéades v 3,13-15, selon lesquelles l'Un, étranger à la multiplicité du logos, se révèle néanmoins dans [la lumière du] Nous comme unité du multiple.

69. D. 30,17 .

70. Ennéades Iv 3,30-31 et 4,3-4. Chez Platon, nous et dianoia ne se distinguent pas par leur objet: les formes intelligibles, mais en ce que celle-ci s'aide de représentations sensibles et ne part pas d'une hypothèse inconditionnée, voir Brisson (1974, 134).

71. P. 2,1,1,275-277. Voir P. 2,1,11,296-297; D. 31,21; 38,7.

72. D. 20,10-11. Voir P. 2,1,39,16-17.

73. D. 31,11 et 33; Narkevics (2006, 9-12). 
l'inspection des empreintes de l'intellect ${ }^{74}{ }^{\prime}$, ne dit pas que le nous soit indissolublement "ratio et imaginatio ${ }^{75}$ ", mais se redéploie sur un mode aristotélicien : "non circonscrit » vaut de l'intellect patient «sans forme », tel une tablette de cire vierge où l'intellect agent produit les formes intelligibles comme la luminosité, les couleurs ${ }^{76}$. L'ascèse noétique consiste justement à nettoyer le nous-miroir des «empreintes» imaginatives d'ici-bas — avant tout ce qui relève de l'ordre du créé - qui entachent et brouillent l'intuition des intelligibles ${ }^{77}$; à le séparer "de ce monde de la matière et de la chair ${ }^{78}$ ». Ainsi rendu diaphane, il brille «des rayons de l'Esprit dans leur puretét $^{79}$ ", et par Lui qui scrute les profondeurs de Dieu, reçoit la gnose théo$\operatorname{logique}^{80}$ de "reflets parfaits ${ }^{81}$ », purement noétiques, voire un aperçu fulgurant de la Vérité: de la Lumière même qui l'éclaire ${ }^{82}$.

Ces épiphanies noétiques conforment le nous aux vertus du Dieu sans forme $^{83}$ et le rendent déjà "plus semblable à la Lumière ${ }^{84}$ ", mimêsis qu'illustre la participation théorétique à l'unité trinitaire ou aux puissances du Verbe. La ré-émanation des rayons de l'Esprit remonte vers sa source selon une "métaphore» - au sens d'un transport vers une similitude et une proximité croissante ${ }^{85}$ - qui fait de la contemplation « la mère de la divine ascension et l'instrument de la divinisation ${ }^{86}{ }$ commencée ici-bas.

Grégoire préfigure en somme sous la forme métaphorique du dialogue avec la Lumière la doctrine byzantine des «énergies » divinisatrices émanant de l'essence divine déjà formulée par son ami Basile ${ }^{87}$ et établit ainsi une

74. P. $1,2,34,26-28$.

75. Contra Børnstein (2006, 53-57).

76. De anima III,4-5. Au v. 26 de notre poème, ou perigraphos pourrait aussi signifier «non inscrit».

77. D. 2,7;12,4 ; P. 2,1,36,8 ; 2,1,13,210-214. Voir déjà Gautier (2006, 207-208).

78. D. 21,2. Voir. D. 2,7; P. 2,1,1,194-197.

79. D. 12,4 .

80. D. 43,65 .

81. P. $2,1,36,10$.

82. Cp. D. 38,7 et Ennéades v 5,7,24 sqq.

83. Cf. Plotin, Ennéades I 2, 2, 21-22, trad. Bréhier : "plus un être participe à la forme [des vertus], plus il devient semblable au divin, qui est sans forme ».

84. D. $28,17$.

85. Cf. T. Th. Tollefsen 2006, 259, mais de la seule divinisation eschatologique et sans rapport avec l'être-à-l'image.

86. D. 3, 1, trad. J. Bernardi, SC 247, 243, cf. D. 2, 7. Cf. Athénagore, Legatio pro christianis 10, éd. Geffcken, 127: le pneuma agissant dans les prophètes, émanation (aporroia) de Dieu, remonte vers sa source comme un rayon de soleil.

87. Cf. N. Russel, 2004, 301-308; V. Lossky 2006, chap. II, IV et XI; supra, n. 81. 
continuité dynamique entre la vision en miroir et le face à face. Par transitivité, le vrai théologien, homme "divin ", est — par l'éclat de sa vie et de sa prédication - lumière pour les autres, «médiateur » entre eux et Dieu selon la définition nazianzène du sacerdoce ${ }^{88}$ :

La tâche du prêtre consiste en cela seul:

Purifier les âmes par la vie et la parole

En les menant vers là-haut par des motions divinement inspirées (entheois), Intellect élevé (hupsinous) et serein, des seuls divins

Reflets sans tache portant l'empreinte,

Formant intérieurement comme un miroir ${ }^{89}$.

\section{Références}

Althaus, H. (1972), Die Heilslehre des heiligen Gregor von Nazianz, Münster, Aschendorf (Münsterische Beiträge zur Theologie, 34).

Armstrong, A. H. (1990a), Étude III : "The Negative Theology of nous in Later Platonism ", Hellenic and Christian Studies, Londres, Aldershot (= H. D. Blume et F. Mann (1983), dir., Platonismus und Christentum. Festschrift füt Heinrich Dörrie, Münster Westfalen, Aschendorf, Jahrbuch für Antike und Christentum, Ergäzungsband 10), p. 31-37. $55,1988)$ p. $147-181$.

(1990b), ibid., Étude vi : «Platonic Mirrors» (= Eranos Jahrbuch, (1990c), ibid., étude Ix: " Pagan and Christian Traditionalism in The First Three Centuries» (= Studia Patristica, xv, 1984), p. 423-432. (1990d), ibid., Étude x: «Philosophy, Theology and Interpretation. The Interpretation of Interpreters » (= Eriugena, Studien zu seinen Quelle. Vorträge des III Internationalen Eriugena-Colloquiums, Heidelberg, C. Winter, 1980), p. 7-14.

BøRTNESs, J. (2006), "Rhetoric and Mental Images in Gregory », dans J. Børtness et T. Hägg, dir., Gregory of Nazianzus. Images and Reflections, Copenhague, Museum Tusculanum Press, p. 37-57.

88. D. 2, 91, trad. J. Bernardi, SC 247, 208 ; P. 2, 1, 1, 129-132; Ep. 79.

89. P. $2,1,12,751-756$. 
BRISSON, L. (1974), Le même et l'autre dans la structure ontologique du Timée de Platon, Paris, Klincksieck.

DieHLe, A. (1983), «Vom sonnhaften Auge und vieless mehr Zustand », dans H. D. Blume et F. Mann, dir., Platonismus und Christentum. Festschrift füt Heinrich Dörrie, Münster Westfalen, Aschendorf, Jahrbuch für Antike und Christentum, Ergäzungsband 10, p. 85-91.

FrÈre, J. (1996), «Fonction représentative et représentation. FANTASIA et FANTASMA selon Aristote », dans G. R. Dherbey, dir., Corps et âme. Sur le De anima d'Aristote, Paris, Vrin (Bibliothèque d'histoire de la philosophie), p. 331-348.

Gallay, P. (1974), Grégoire de Nazianze, Lettres théologiques. Introduction, texte critique, traduction et notes, Paris, Cerf (Sources Chrétiennes, 208).

Gautier, F. (2002), La retraite et le retour au monde chez Grégoire de Nazianze, Turnhout, Brepols (Bibliothèque de l'École des Hautes Études, 114).

2006, "Grégoire de Nazianze. La théologie, de la raison à l'Esprit ", dans E. Moutsopoulos et G. Lekkas, dir., La transcendance dans la philosophie grecque tardive et dans la pensée chrétienne. Actes du VI ${ }^{e}$ congrès de philosophie grecque, Athènes, 22-27 septembre 2004, Paris, Vrin (Centre international d'études platoniciennes et aristotéliciennes, Série Recherches, 5), p. 197-209.

Hoffmann, P. (1997), "L'expression de l'indicible dans le néoplatonisme grec, de Plotin à Damascius », dans C. Lévy et L. Pernot, dir., Dire l'évidence, Paris/Montréal, L'Harmattan (Cahiers de philosophie de l’Université Paris XII — Val-de-Marne), p. 335-391.

Labarrière, J.-L. (2004), «Phantasia », dans B. Cassin, dir., Vocabulaire européen des philosophies. Dictionnaire des intraduisibles, Paris, Seuil (Le Robert), p. 931-935.

Le Boulluec, A. (2006), Alexandrie antique et chrétienne. Clément et Origène (Études Augustiniennes, Série Antiquités, 178).

Lossky, V. (2006³) [1944], Essai sur la théologie mystique de l'Église d'Orient, Paris, Cerf.

McGuckin, J. (2001), Saint Gregory of Nazianzus. An Intellectual Biography, New York, St Vladimir Seminary Press.

Merker, A. (2003), La vision chez Platon et Aristote, Sankt Augustin, Academia Verlag (International Plato Studies, 16), p. 24-39. 
Moreschini, C. (1997), Filosofia e letteratura in Gregorio di Nazanzianzo, Milan, Centro di Ricerche di Metafisica Vita e Pensiero.

Mugler, C. (1964), Dictionnaire historique de la terminologie optique des Grecs, Paris, Klinksieck.

NARKeVICS, E. (2006), "Skiagraphia: Outlining the conception of God in Gregory's Theological Orations », dans J. Børtness et T. Hägg, dir., Gregory of Nazianzus. Images and Reflections, Copenhague, Museum Tusculanum Press, p. 83-112.

Norris, F. W. (1991), Faith Gives Fullness to Reasoning: The Five Theological Orations of Gregory Nazianzen. Intr. et com. F. W. Norris, trad. L. Wickham et F. Wiliams, Leyden, Brill (Vigiliæ Christianæ, Suppl. 13).

Philon D'Alexandrie (1963), De vita contemplativa 78, trad. P. Miquel, Paris, Cerf (Les œuvres de Philon d'Alexandrie, 29).

Richard, A. (2003), Cosmologie et théologie chez Grégoire de Nazianze, Institut d'Études Augustiniennes, Paris (Études Augustiniennes, Série Antiquité, 169).

ROUVERET, A. (1989), Histoire et imaginaire de la peinture ancienne : $v^{e}$ siècle av. J. C. - Ir siècle ap. J. C., Rome, École française de Rome (Bibliothéque des Écoles françaises d'Athènes et de Rome 274).

Russel, N. (2004), The Doctrine of Deification in the Greek Patristic Tradition, Oxford, Oxford University Press (Oxford Early Christian Studies).

Schroeder, F. M. et R. B. Todd (1990), Two Greek Aristotelian Commentators on The Intellect. The De Intellectu attributed to Alexander of Aphrodisias and Themistius' Paraphrase of Aristotle De Anima 3.4-8, Toronto, Pontifical Institute for Mediaeval Studies.

Simon, G. (1988), Le Regard, l'être et l'apparence dans l'optique de l'Antiquité, Paris, Seuil. (2004), «Eidôlon", dans B. Cassin, dir., Vocabulaire européen des philosophies. Dictionnaire des intraduisibles, Paris, Seuil (Le Robert), p. 335-340.

Szymusiak, I. (1966), "Grégoire de Nazianze et le péché », Studia Patristica, 9, p. 288-305.

Tollefsen, T. T. (2006), "Theosis according to Gregory », dans J. Børtness et T. Hägg, dir., Gregory of Nazianzus. Images and Reflections, Copenhague, Museum Tusculanum Press, p. 257-270. 
Vasiluu, A. (2004), «Diaphane », dans B. Cassin, dir., Vocabulaire européen des philosophies. Dictionnaire des intraduisibles, Paris, Seuil (Le Robert), p. 307-312.

(1997), Du diaphane. Image, milieu, lumière dans la pensée antique et médiévale, Paris, Vrin (Études de philosophie médiévale).

Winslow, D.F. (1979), The Dynamics of Salvation. A study in Gregory of Nazianzus, Philadephie, The Philadelphia Patristic Foundation (Patristic Monograph Series, 7).

\section{Résumé}

Grégoire de Nazianze, le plus affirmatif des Cappadociens quant à la divinisation de l'homme dès cette vie, semble logiquement minorer le fossé entre la vision de Dieu «comme en un miroir» ici-bas et le «face à face " promis (1 Co 13,12). Cet article examine ce fait par l'analyse des métaphores optiques utilisées par Grégoire pour les relations entre la «Lumière» ou l'Intellect divin et son "image ", l'homme, aux différentes étapes de l'économie du salut. Le Nazianzène situe la relation de l'individu à Dieu, dynamique et assimilative, entre la «Lumière »/Intelligence divine et son reflet émané en nous. Leur union en Christ rétablit à travers l'illumination baptismale la translucidité adamique perdue, rouvrant à l'individu le passage de la vision "en miroir» d'ici-bas de sa source, l'essence divine, à l'illumination plénière du «face à face » divinisateur. L'imagerie nazianzène du « dialogue » contemplatif entre le nous ou «lumière » humain(e) et Dieu, quelle que soit l'insistance sur la transcendance du second à l'égard du visible et sur les attaches du premier au sensible, implique réellement pour le nous contemplatif une communication avec, et une assimilation à la source dont il émana. L'intellect-miroir du contemplatif, à condition d'un «nettoyage » portant à la perfection noétique les images reflétant Dieu, peut déjà en former un aperçu grâce à la lumière de l'Esprit, en recevoir les «énergies ", et servir de médiateur sacerdotal entre la Lumière et les autres individus.

\section{Abstract}

Gregory of Nazianzus, the most assertive Cappadocian Father concerning man's deification as of this life, seems to undermine the gap between the earthly vision of God "as if in a mirror " and the hoped-for "face to face" in the afterlife (1 Cor $12: 13$ ). This paper scrutinizes this fact by focusing on the optical metaphors used by Gregory to depict relations between the 
divine Intellect or "Light» and man as His image during the different stages of the economy of salvation. For Gregory of Nazianzus, the relation of the individual to God is dynamic, integrating the divine "Light»/Intelligence and His emanated reflection in us. Their union in Christ through baptismal illumination restores the lost adamic transparency, so that the individual may advance from the earthly vision "as in a mirror " of the divine essence, to the deifying "face to face». Gregory's imagery of the contemplative "dialogue» between man's nous or «light» and God, for all his insistence on the latter's transcendence towards the visible and on the former's bonds with the senses, implies for the contemplating nous a real communication with, and assimilation to, the divine source from which it originally emanated and towards which he goes back up. The contemplative's intellect-mirror, provided the images reflecting God are brought to their noetic perfection by «cleaning", can form a preview of Him - thanks to the Spirit's light, receive his "energies", and mediate as a priest between the Light and the other individuals. 